\title{
Simulation and design of the HT-KZ Ultra-high energy cosmic rays detector system for cosmic rays with energies above $10^{17} \mathrm{eV}$
}

\author{
A. Duspayev', T. Beremkulov, D. Beznosko, A. lakovlev, K. Yelshibekov, M. \\ Yessenov \\ Nazarbayev University \\ 53 Kabanbai Batyr ave, Astana, KZ \\ E-mail: alisher.duspayev@nu.edu.kz
}

\section{R. U. Beisembaev, V. V. Zhukov}

P. N. Lebedev Physical Institute of the Russian Academy of Sciences

53 Leninskiy Prospekt, Moscow, Russia

In the field of High Energy Physics today there are several open topics left. The Higgs boson has been recently discovered, neutrino oscillations are being studied, and some hints of the dark matter have been detected as well. Another remaining mystery is the origin and the nature of the Ultra-High Energy Cosmic Rays.

There is an active project at Nazarbayev University to construct the HorizonT-Kazakhstan detector system in collaboration with the Tien Shan high-altitude Science Station (TSHSS), a part of Lebedev Physical Institute of the Russian Academy of Sciences. The full R\&D is underway. A significant part of this process is the simulation, testing and construction of individual particle detectors due to the requirements of robustness and high linear range of such detectors combined with low cost and long-term operations with minimal maintenance.

In this paper, the latest results of the simulation activities and experiment testing of different detection components as applicable to the HorizonT-Kazakhstan requirements are presented.

38th International Conference on High Energy Physics

3-10 August 2016

Chicago, USA

\section{${ }^{1}$ Speaker}




\section{Introduction}

The HorizonT-Kazakhstan (HT-KZ) is a distributed detector system under construction at Nazarbayev University (NU), Astana, Kazakhstan. The main purpose of the system is to study the origin and the nature of Ultra-High Cosmic Rays (UHECR) with energies above $\sim 10^{17} \mathrm{eV}$ by analyzing Extensive Air Shower (EAS) signals. EAS occur as the primary particle interacts with the atmosphere. HT-KZ construction is executed in collaboration with Tien Shan high-altitude Science Station, a part of Lebedev Physical Institute of the Russian Academy of Sciences, where the Horizon-T (HT) [1], [2] detector system, the predecessor to HT-KZ, operates currently.

HT-KZ is meant to further investigate properties of UHECR such as arrival anisotropy and multimodal EAS originating from potential new particle type. The phenomenon of multimodal EAS is being studied by the HT [2]. Main motivations for the HT-KZ construction is to study in detail findings from HT to find the multimodal EAS source (a decay of the unknown heavy particle into several UHECR, existence of the exotic sources, e.g., dark matter particle decay).

CORSIKA[3] simulations of the propagation of EAS at such energy in the atmosphere show that the charged particles from the vertical EAS at the distance of $\sim 100 \mathrm{~m}$ from the core pass the observation level in $\sim 15-20 \mathrm{~ns}$, and even faster near the core. Fast detectors and DAQ with a few ns resolution is required.

\section{General description of the HT-KZ system}

The previous report about the status of the project's development is in [4]. The HT-KZ system will consist of eight modules to be distributed on the roofs of NU. Each independent module will consist of 3 plastic scintillator [5] particle detectors (SD) oriented perpendicular to each other. Such geometry has been used in the HT system for the angle measurement of the incident EAS near the horizon. A possibility for liquid scintillator use has been considered in [6]. Each SD will consist of two parts: fast scintillator base (shape of the base has been chosen according to the recent simulation results) and Hamamatsu [7] PMT (Photo Multiplier Tube). Each module will have time resolution $\sim 1.5 \mathrm{~ns}$. About 1 event $/ \mathrm{km}^{2}$ per day $(\sim 1000$ events/year $)$ at primary particle energy of $10^{17} \mathrm{eV}$ is expected with distance between modules $\sim 150 \mathrm{~m}$. Each module is expected to collect data at rate $<200 \mathrm{kbyte} / \mathrm{s}$.

Trigger level and logic of the system will be software controlled using CAEN [8] DT 5743 ADC. Contrary to HT that uses long cables [9], we plan to place ADC at each detection point. Data synchronization and analysis requires time resolution at ns resolution for better determination of the EAS structure and direction to the origin. Simulation of an individual module's operational process and measurements of the PMT linearity range have been done recently.

The simulation of the detector module has been done using Python [10] programming language and ROOT [11] framework. The main purpose of the simulation is to determine the most appropriate geometry of a single detector module taking into account different options for detection medium (glass, Eljen [12] Cerenkov plastic or scintillator), its dimensions and shape (circle or square), PMT placements options (edge, centered front or back sides of the detection medium), possible air waveguide between medium and PMT, uniformity of the Cerenkov photons detection's distribution, width of the signal from the detected particle. 


\section{Simulation of the individual detector module}

According to the results of the simulation, the circular-shaped Cerenkov plastic $(50 \mathrm{~cm}$ radius, $5 \mathrm{~cm}$ width) with back side painted has been chosen as detection medium, PMT is placed on the front side at $1 \mathrm{~m}$ distance from the detection medium (diameter of the scintillation disk). White painting of the back side prevents large losses of light due to escape or absorption of

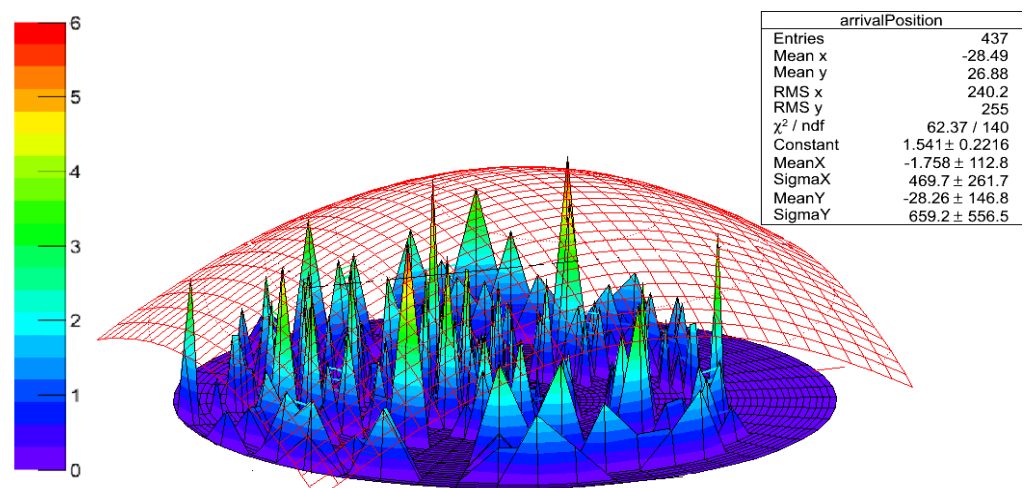

Figure 1: Simulation results for the distribution of the detected photons for the circular-shaped Cereknov plastic

Cerenkov photons coming from the side opposite to PMT placement reflected from a diffused paint ( Figure 1). Such geometry gives better photon production and allows to achieve more uniform and efficient detection probability than other considered options.

\section{PMT linearity response testing}

Non-linearity measurements have been conducted using PMT signals within the CAEN DT5743/DT5730 ADC detection range up to $2 \mathrm{~V}$. Experimental setup used for the measurement is shown in Figure 2 (left). It consists of the PMT, light source (LED) and the PIN diode for the LED light level monitoring. LED and PIN were placed in a small black box during the experiment with a pinhole in a side serving as a light source for a PMT.
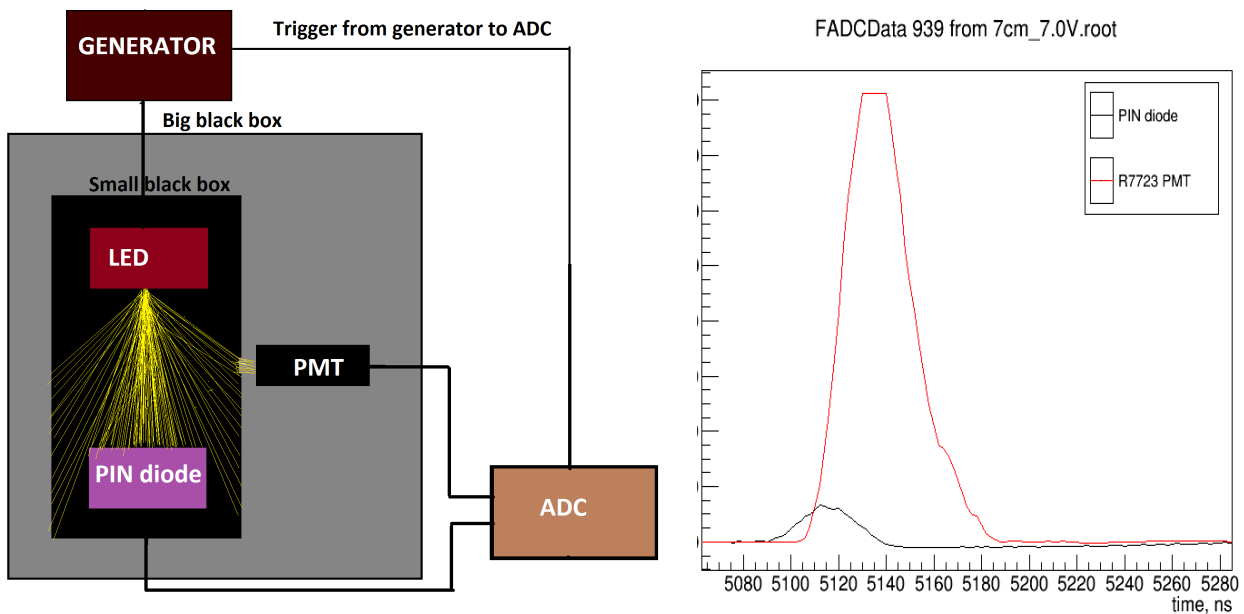

Figure 2: Experimental setup of the nonlinearity response testing (left) and PMT pulse from ADC (right) 
Width of function generator pulse was chosen to mimic the realistic data signal. Pulse amplitude was varied between $5 \mathrm{~V}$ and $10 \mathrm{~V}$; the PMT was placed at $7 \mathrm{~cm}$ and $22 \mathrm{~cm}$ from the pinhole. This keeps PMT output below 2 V. Pulses from PMT and PIN are integrated and the areas are used for the analysis. The plot of PIN signal area vs. PMT signal area is shown in Figure 3.

The PIN diode is linear over entire light intensity range used. The non-linearity is observed only for the PMT pulses exceeding ADC range as in Figure 2 (right), indicated by the two last points in Figure 3. PMT response is linear over the entire ADC range.

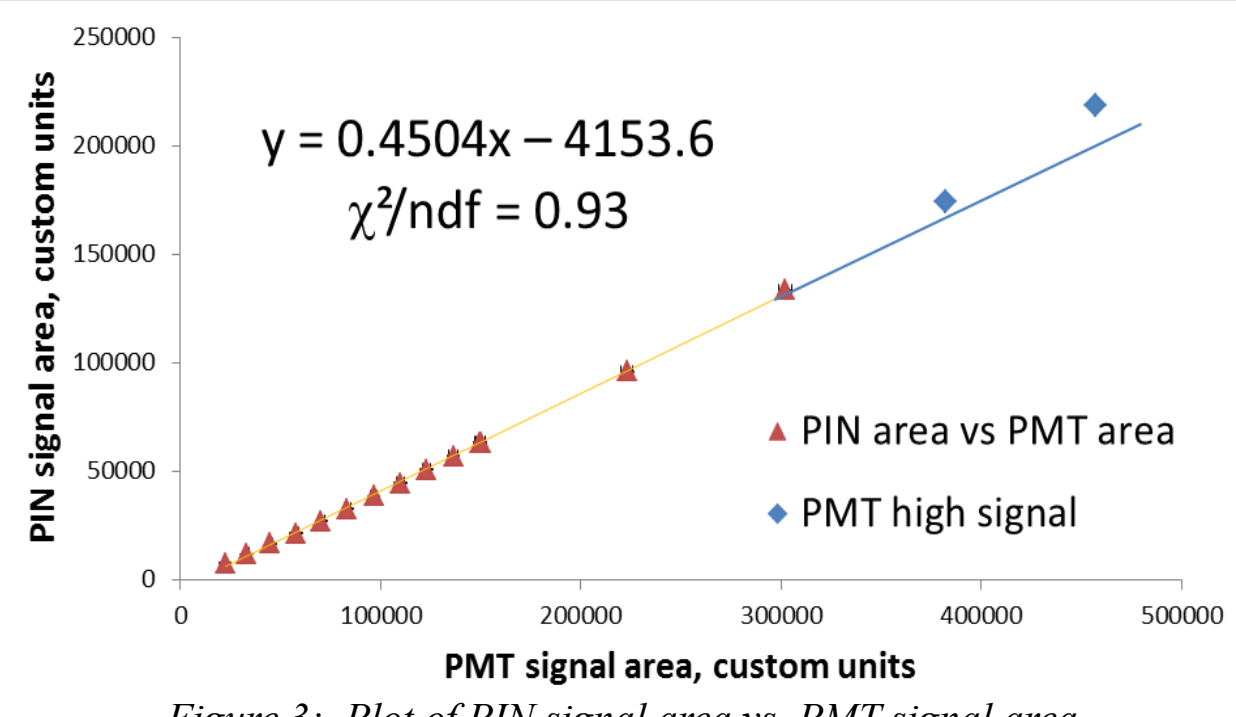

Figure 3: Plot of PIN signal area vs. PMT signal area

\section{Conclusion}

The HT-KZ system is a new EAS detector system. Simulation of the individual detector module's performance and PMT linearity measurements have been done. Circular shape of the scintillator base and the distance between PMT and the detector base are chosen according to the results of the simulation. Linearity measurements of Hamamatsu R7723 PMT showed that its response is linear over the entire ADC range. Pulse synchronization and data analysis software R\&D is in the process at NU. Construction and installation of the first prototype of the HT-KZ system will be done in the nearest future. 


\section{References}

[1] R.U. Beisembaev et al., The 'Horizon-T' Experiment: Extensive Air Showers Detection, arXiv:1605.05179 [physics.ins-det], (2016)

[2] D. Beznosko et al., Horizon-T extensive air showers detector system operations and performance, in proceedings to ICHEP-2016 conference POS (ICHEP2016) 784

[3] D. Heck et al., CORSIKA: A Monte Carlo Code to Simulate Extensive Air Showers, Forschungszentrum Karlsruhe Report FZKA (6019)

[4] A. Duspayev at al., The distributed particle detectors and data acquisition modules for Extensive Air Shower measurements at "Horizon-T KZ" experiment, in proceedings to PhotoDet2015 conference PoS (PhotoDet20155) 06

[5] A Baitenov et al., Technical manual: a survey of scintillating medium for high-energy particle detection, arXiv:1601.00086, (2016)

[6] L. J. Bignell et al., Characterization and Modeling of a Water-based Liquid Scintillator, Journal of Instrumentation, Vol. 10, p 12009, IOP Publishing (2015)

[7] Hamamasu Photonics K.K., Electron Tube Division, 314-5, Shimokanzo, Iwata City, Shizuoka Pref., 438-0193, Japan, http://www.hamamatsu.com

[8] CAEN S.p.A. Via della Vetraia, 11, 55049 Viareggio Lucca, Italy. http://caen.it.

[9] T Beremkulov et al., Horizon-T Experiment Calibrations-Cables, arXiv:1608.04312, 2016/8/15

[10] G. van Rossum, Python tutorial, Technical Report CS-R9526, Centrum voor Wiskunde en Informatica, Amsterdam, (1995)

[11] R. Brun, F. Rademakers, ROOT: An object oriented data analysis framework, Nucl. Instrum. Meth. A, Vol. 389, p. 81-86 (1997)

[12] Eljen Technology. 1300 W. Broadway, Sweetwater, Texas 79556, United States 